\title{
ISOMETRIES BETWEEN MATRIX ALGEBRAS
}

\author{
WAI-SHUN CHEUNG, CHI-KWONG LI and YIU-TUNG POON
}

(Received 2 October 2001; revised 21 March 2003)

Communicated by G. Willis

\begin{abstract}
As an attempt to understand linear isometries between $C^{*}$-algebras without the surjectivity assumption, we study linear isometries between matrix algebras. Denote by $M_{m}$ the algebra of $m \times m$ complex matrices. If $k \geq n$ and $\phi: M_{n} \rightarrow M_{k}$ has the form $X \mapsto U[X \oplus f(X)] V$ or $X \mapsto U\left[X^{\prime} \oplus f(X)\right] V$ for some unitary $U, V \in M_{k}$ and contractive linear map $f: M_{n} \rightarrow M_{k}$, then $\|\phi(X)\|=\|X\|$ for all $X \in M_{n}$. We prove that the converse is true if $k \leq 2 n-1$, and the converse may fail if $k \geq 2 n$. Related results and questions involving positive linear maps and the numerical range are discussed.
\end{abstract}

2000 Mathematics subject classification: primary 15A04, 15A60.

Keywords and phrases: isometry, matrices, linear maps.

\section{Introduction}

In [6], Kadison characterized surjective linear isometries on $C^{*}$-algebras. The problem without surjectivity seems very difficult even in the finite dimensional case. In this paper, we study linear isometries from $M_{n}$ to $M_{k}$, that is, linear maps $\phi: M_{n} \rightarrow M_{k}$ such that $\|\phi(A)\|=\|A\|$ for all $A \in M_{n}$, where $M_{m}$ is the algebra of $m \times m$ complex matrices and $\|\cdot\|$ is the spectral norm. Clearly, if such a linear isometry $\phi$ exists, then $k \geq n$. If $k=n$, it follows from the result of Kadison [6] that $\phi$ has the form $X \mapsto U X V$ or $X \mapsto U X^{\prime} V$, for some unitary $U, V \in M_{n}$. One can modify the above maps to norm preserving linear maps $\phi: M_{n} \rightarrow M_{k}$ with $k>n$, namely, if $U, V \in M_{k}$ are unitary and $f: M_{n} \rightarrow M_{k-n}$ is a contractive linear map, then $\phi: M_{n} \rightarrow M_{k}$ defined by

$$
X \mapsto U[X \oplus f(X)] V \quad \text { or } \quad X \mapsto U\left[X^{\prime} \oplus f(X)\right] V
$$

(C) 2004 Australian Mathematical Society 1446-7887/04 $\$$ A2.00+0.00 
is a linear isometry. It is natural to ask whether the converse of this statement holds. We have the following result.

TheOREM 1.1. Suppose $k \leq 2 n-1$, and $\phi: M_{n} \rightarrow M_{k}$ is linear such that $\|\phi(X)\|=\|X\|$ for all $X \in M_{n}$. Then $k \geq n$, and there exist $U, V \in M_{k}$ and $a$ contractive linear map $f: M_{n} \rightarrow M_{k-n}$ such that $\phi$ has the form

$$
X \mapsto U[X \oplus f(X)] V \quad \text { or } \quad X \mapsto U\left[X^{t} \oplus f(X)\right] V
$$

Moreover, if $k \geq 2 n \geq 4$, then there exists a norm preserving linear map $\psi: M_{n} \rightarrow M_{k}$ that is not of the form (1.1).

Recall that $B \in M_{n}$ is essentially Hermitian if $B=a A+b I$ for some Hermitian $A$ and $a, b \in \mathbb{C}$, equivalently, $B$ is normal and its eigenvalues lie on a straight line. It turns out that Theorem 1.1 can be deduced from the following result concerning unital linear maps $\phi: M_{n} \rightarrow M_{k}$ that preserve the norm of essentially Hermitian matrices.

THEOREM 1.2. Suppose $k \leq 2 n-2$, and $\phi: M_{n} \rightarrow M_{k}$ is a linear map. Then $\phi$ satisfies $\phi\left(I_{n}\right)=I_{k}$ and $\|\phi(X)\|=\|X\|$ for all essentially Hermitian matrices $X \in M_{n}$ if and only if $k \geq n$, and there exist a unitary $U \in M_{k}$ and a unital positive linear map $f: M_{n} \rightarrow M_{k-n}$ such that $\phi$ has the form

$$
X \mapsto U[X \oplus f(X)] U^{*} \quad \text { or } \quad X \mapsto U\left[X^{t} \oplus f(X)\right] U^{*}
$$

Moreover, if $k \geq 2 n-1 \geq 3$, then there exists a linear map $\psi: M_{n} \rightarrow M_{k}$ which is not of the form (1.2) but satisfies $\psi\left(I_{n}\right)=I_{k}$ and $\|\psi(X)\|=\|X\|$ for all essentially Hermitian matrices $X \in M_{n}$.

We prove some auxiliary results in the next section, and give the proofs of Theorems 1.1 and 1.2 in Section 3. Some related results and questions are discussed in the last section.

In our discussion, we let $\left\{e_{1}, \ldots, e_{n}\right\}$ be the standard basis for $\mathbb{C}^{n}$, and $E_{i j}=e_{i} e_{j}^{t}$ be the standard matrix unit. Denote by $\mathscr{H}_{n}$ the real linear space of $n \times n$ Hermitian matrices, and $\lambda_{1}(A) \geq \cdots \geq \lambda_{n}(A)$ the eigenvalues of $A \in \mathscr{H}_{n}$; we write $A>0$ if $\lambda_{n}(A)>0$ and $A \geq 0$ if $\lambda_{n}(A) \geq 0$.

\section{Auxiliary results}

THEOREM 2.1. Suppose $\phi: M_{n} \rightarrow M_{k}$ satisfies $\|\phi(A)\| \leq\|A\|$ for all essentially Hermitian $A \in M_{n}$ and $U^{*} \phi\left(I_{n}\right) V=I_{p} \oplus D$, where $U, V \in M_{k}$ are unitary and $D \in M_{k-p}$ is a diagonal matrix with diagonal entries in the interval $[0,1)$. Use the 
first $p$ columns of $U$ (respectively $V$ ) to form the matrix $U_{1}$ (respectively $V_{1}$ ). Then the mapping $\psi: M_{n} \rightarrow M_{p}$ defined by $\psi(X)=U_{1}^{*} \phi(X) V_{1}$ satisfies the following conditions:

(1) $\psi\left(I_{n}\right)=I_{p}$.

(2) $\|\psi(A)\| \leq\|A\|$ for all essentially Hermitian $A \in M_{n}$.

(3) $\psi(A) \geq 0$ for all $A \geq 0$.

(4) $\psi\left(A^{*}\right)=\psi(A)^{*}$ for all $A \in M_{n}$.

If, in addition, $\|\phi(A)\|=\|A\|$ for all $A \in \mathscr{H}_{n}$, then

(5) $\|\psi(B)\|=\|B\|$ for all essentially Hermitian $B \in M_{n}$.

(6) $\psi(A) \geq 0$ if and only if $A \geq 0$.

(7) For every $A \in \mathscr{H}_{n}, \lambda_{1}(A)=\lambda_{1}(\psi(A))$ and $\lambda_{n}(A)=\lambda_{p}(\psi(A))$.

PROOF. Conditions (1) and (2) follow from the definition.

For (3), suppose $A \geq 0$ and $\psi(A)=B+i C, B, C \in \mathscr{H}_{p}$. For any unit vector $x \in \mathbb{C}^{p}$, let $b=x^{*} B x$ and $c=x^{*} C x$. We are going to prove that $c=0$. It will then follow that $C=0$. To prove our claim, for each positive integer $m$, let $A_{m}=A-b I_{n}+i\left(m c I_{n}\right)$. Then

$$
\begin{aligned}
\left\|A-b I_{n}\right\|^{2}+m^{2} c^{2} & \geq\left\|\left(A-b I_{n}\right)^{2}+m^{2} c^{2} I_{n}\right\|=\left\|A_{m} A_{m}^{*}\right\|=\left\|A_{m}\right\|^{2} \\
& \geq\left\|\psi\left(A_{m}\right)\right\|^{2} \geq\left|x^{*} \psi\left(A_{m}\right) x\right|^{2} \\
& =\left|x^{*}\left(B-b I_{n}+i\left(m c I_{n}+C\right)\right) x\right|^{2} \\
& =\left|x^{*} B x-b+i\left(m c+x^{*} C x\right)\right|^{2}=|(m+1) c|^{2} .
\end{aligned}
$$

Hence, $c=0$ as asserted. So, $\psi(A)=B$. If $s>0$ is small, then

$$
\left\|I_{p}-s B\right\|=\left\|\psi\left(I_{n}-s A\right)\right\| \leq\left\|I_{n}-s A\right\| \leq 1 .
$$

Therefore, $B \geq 0$.

Condition (4) follows readily from (3).

Now, suppose that $\|\phi(A)\|=\|A\|$ for all $A \in \mathscr{H}_{n}$. Let $B \in M_{n}$ be essentially Hermitian, that is, $B=a A+b I$ for some $A \in \mathscr{H}_{n}$ and $a, b \in \mathbb{C}$. We are going to show that $\|\psi(B)\|=\|B\|$. The claim clearly holds if $a=0$. So, without loss of generality, we assume that $a=1$.

First, consider the case when $b=0$. Hence, $B=A \in \mathscr{H}_{n}$. We may further assume that $\|A\|=\lambda_{1}(A)$; otherwise, replace $A$ by $-A$. Then

$$
\begin{aligned}
1+\lambda_{1}(A) & =\left\|I_{n}+A\right\|=\left\|\phi\left(I_{n}+A\right)\right\|=\left\|U^{*} \phi\left(I_{n}+A\right) V\right\| \\
& =\left\|\left(I_{p} \oplus D\right)+U^{*} \phi(A) V\right\| .
\end{aligned}
$$


So there exist unit vectors $x$ and $y$ in $\mathbb{C}^{k}$ such that

$$
\begin{aligned}
1+\lambda_{1}(A) & =y^{*}\left[\left(I_{p} \oplus D\right)+U^{*} \phi(A) V\right] x \\
& \leq\left|y^{*}\left(I_{p} \oplus D\right) x\right|+\left|y^{*} U^{*} \phi(A) V x\right| \leq 1+\lambda_{1}(A) .
\end{aligned}
$$

Therefore, $y=x=\left(I_{p} \oplus D\right) x$ and $U^{*} \phi(A) V x=\lambda_{1}(A) x$. Hence, $x=\left[\begin{array}{c}x_{1} \\ 0\end{array}\right]$, where $x_{1} \in \mathbb{C}^{p}$ and $\psi(A) x_{1}=\lambda_{1}(A) x_{1}$. As a result, $\|\psi(A)\|=\|A\|$.

For the general case, suppose $B=A+(a+i b) I_{n}$, where $A \in \mathscr{H}_{n}$ and $a, b \in \mathbb{R}$. Then

$$
\begin{aligned}
\|\psi(B)\|^{2} & =\left\|\psi\left(A+a I_{n}\right)+i b I_{p}\right\|^{2}=\left\|\psi\left(A+a I_{n}\right)\right\|^{2}+|b|^{2} \\
& =\left\|A+a I_{n}\right\|^{2}+|b|^{2}=\left\|\left(A+a I_{n}\right)+i b I_{n}\right\|^{2}=\|B\|^{2} .
\end{aligned}
$$

This proves (5).

For (6), let $A \in M_{n}$ such that $\psi(A) \geq 0$. Let $A=B+i C$ where $B, C \in \mathscr{H}_{n}$. Then by (4), we have $\psi(B-i C)=\psi\left(A^{*}\right)=\psi(A)^{*}=\psi(A)$. Hence, $\psi(C)=0$ implies $C=0$, that is, $A \in \mathscr{H}_{n}$. For every $t>\|\psi(A)\|$, we have $t \geq\left\|t I_{p}-\psi(A)\right\|=$ $\left\|t I_{n}-A\right\|$. Therefore, $A \geq 0$.

For (7), let $A \in \mathscr{H}_{n}$ and $t \in \mathbb{R}$. By (6), we have

$$
t \geq \lambda_{1}(A) \Longleftrightarrow t I_{n}-A \geq 0 \Longleftrightarrow t I_{p}-\psi(A) \geq 0 \Longleftrightarrow t \geq \lambda_{1}(\psi(A)) .
$$

Therefore, $\lambda_{1}(A)=\lambda_{1}(\psi(A))$. Similarly, $\lambda_{n}(A)=\lambda_{p}(\psi(A))$.

REMARK 2.2. Note that one cannot weaken the hypothesis in Theorem 2.1 to $\|\phi(A)\| \leq\|A\|$ for all Hermitian $A \in M_{n}$. For example, suppose $\phi: M_{2} \rightarrow M_{3}$ is given by

$$
\phi(A)=A \oplus[(a+d+i(a-d)) / 2] \quad \text { if } \quad A=\left(\begin{array}{ll}
a & b \\
c & d
\end{array}\right)
$$

Then $\phi\left(I_{2}\right)=I_{3}$ and $\|\phi(A)\|=\|A\|$ for all $A \in \mathscr{H}_{2}$. However, if $A=[1] \oplus 0 \in \mathscr{H}_{2}$, then $\phi(A)=A \oplus[(1+i) / 2] \notin \mathscr{H}_{3}$ and

$$
\left\|\phi\left(2 A+2 i I_{2}\right)\right\|=\left\|\left(2 A+2 i I_{2}\right) \oplus[1+3 i]\right\|=\sqrt{10}>\sqrt{8}=\left\|2 A+2 i I_{2}\right\| .
$$

In fact, none of the conditions (2)-(7) holds.

Note also that the only place where we use the condition $\|\phi(A)\| \leq\|A\|$ for all essentially Hermitian $A \in M_{n}$ is in showing that $\psi(A) \in \mathscr{H}_{k}$ for all $A \in \mathscr{H}_{n}$. Hence, the proof of Theorem 2.1 also gives the equivalence of (a)-(c) in the following theorem. 
THEOREM 2.3. Suppose $k \leq 2 n-2$, and $\phi: \mathscr{H}_{n} \rightarrow \mathscr{H}_{k}$ is a linear map satisfying $\phi\left(I_{n}\right)=I_{k}$. The following conditions are equivalent.

(a) $\|\phi(X)\|=\|X\|$ for all $X \in \mathscr{H}_{n}$.

(b) $A \in \mathscr{H}_{n}$ is positive semidefinite if and only if $\phi(A)$ is positive semidefinite.

(c) For every $A \in \mathscr{H}_{n}, \lambda_{1}(A)=\lambda_{1}(\phi(A))$ and $\lambda_{n}(A)=\lambda_{k}(\phi(A))$.

(d) We have $k \geq n$, and there exist a unitary $U \in M_{k}$ and a unital positive linear map $f: \mathscr{H}_{n} \rightarrow \mathscr{H}_{k-n}$ such that $\phi$ has the form

$$
X \mapsto U[X \oplus f(X)] U^{*} \quad \text { or } \quad X \mapsto U\left[X^{t} \oplus f(X)\right] U^{*}
$$

PROOF. By the discussion before the theorem, we see that (a), (b), (c) are equivalent. It is clear that (d) implies all the conditions (a)-(c). In the following, we assume that one, and hence all, of the conditions (a)-(c) holds, and prove condition (d) by induction on $n \geq 2$. By (a), we have $k \geq n$.

Suppose $n=k$. If $X_{1} \in \mathscr{H}_{n}$ is a rank one orthogonal projection, then there exist rank one orthogonal projections $X_{2}, \ldots, X_{n}$ such that $\sum_{j=1}^{n} X_{i}=I_{n}$. By condition (c), $\phi\left(X_{j}\right)$ is positive semi-definite with largest eigenvalue equal to one for $j=1, \ldots, n$. Moreover,

$$
\sum_{j=1} \operatorname{tr} \phi\left(X_{j}\right)=\operatorname{tr} \phi\left(\sum_{j=1}^{n} X_{j}\right)=\operatorname{tr} I_{n}=n .
$$

Thus, $\phi\left(X_{j}\right)$ has eigenvalues $1,0, \ldots, 0$, that is, $\phi\left(X_{j}\right)$ is a rank one orthogonal projection, for $j=1, \ldots, n$. Hence, $\phi$ maps rank one orthogonal projections to rank one orthogonal projections. By [3, Theorem 3], we conclude that there exists a unitary $S \in M_{n}$ such that $\phi$ has the form

$$
X \mapsto S X S^{*} \quad \text { or } \quad X \mapsto S X^{t} S^{*}
$$

Thus, condition (d) holds if $n=k$. Note that if $n=2$, then $n \leq k \leq 2 n-2$ implies that $n=k=2$. So, condition (d) holds. Now, suppose $n \geq 3$ and $n<k \leq 2 n-2$, and the result is true for linear maps from $\mathscr{H}_{r}$ to $\mathscr{H}_{s}$ for any $r<n$ and $s \leq 2 r-2$. We shall establish the following.

Claim. There exist unitary matrices $V \in M_{n}$ and $U \in M_{k}$ such that the mapping

$$
A \mapsto U \phi\left(V A V^{*}\right) U^{*}
$$

has the form

$$
X \mapsto g(X) \oplus \tilde{f}(X)
$$

where $\tilde{f}: \mathscr{H}_{n} \rightarrow \mathscr{H}_{k-n}$ is a unital positive linear map, and $g: \mathscr{H}_{n} \rightarrow \mathscr{H}_{n}$ is unital, linear, and maps rank one orthogonal projections to rank one orthogonal projections. 
Once the claim is proved, we can apply [3, Theorem 3] to $g$ and conclude that $g$ has the form (2.1) for some unitary $S \in M_{n}$. Consequently, the original map $\phi$ will satisfy condition (d).

Note that we only need to show that there exist unitary matrices $U$ and $V$ such that the mapping in (2.2) is a direct sum of two linear maps in the form (2.3). It will then follow (say, from (b)) that $\tilde{f}$ is a unital positive linear map as asserted.

We establish several assertions to prove our claim.

ASSERTION 1. For each $j \in\{1, \ldots, n\}, \phi\left(E_{j j}\right)$ has largest and smallest eigenvalues equal to 1 and 0 , respectively. Moreover, if $v \in \mathbb{C}^{k}$ is a unit vector such that $v^{*} \phi\left(E_{j j}\right) v=1$, then $v^{*} \phi(X) v=0$ for any $X \in \mathscr{H}_{n}$ with $(j, j)$ entry equal to 0 .

Proof. The first statement follows from (c). To prove the second statement, we may assume that $j=1$. Suppose $v \in \mathbb{C}^{n}$ is a unit vector such that $v^{*} \phi\left(E_{11}\right) v=1$. If $Y=[0] \oplus Y_{1}$ with $Y_{1} \in \mathscr{H}_{n-1}$, then for any $t \in[-1,1]$,

$$
v^{*}\left[\phi\left(E_{11}\right)+t \phi(Y)\right] v \leq\left\|E_{11}+t Y\right\|=1 .
$$

Thus, $v^{*} \phi(Y) v=0$. If $Z=e_{1} z^{*}+z e_{1}^{*}$ for some unit vector $z \in \operatorname{span}\left\{e_{2}, \ldots, e_{n}\right\}$, then there exists a unitary matrix $U=[1] \oplus U_{1}$ with $U_{1} \in M_{k-1}$ such that $U Z U^{*}=$ $E_{12}+E_{21}$. Therefore, for every $t \in[-1,1]$,

$$
\begin{aligned}
v^{*}\left[\phi\left(E_{11}\right)+t \phi(Z)\right] v & \leq\left\|E_{11}+t Z\right\|=\left\|U\left(E_{11}+t Z\right) U^{*}\right\| \\
& =\left\|E_{11}+t\left(E_{12}+E_{21}\right)\right\| \leq \sqrt{1+2 t^{2}} .
\end{aligned}
$$

Again, we have $v^{*} \phi(Z) v=0$. Consequently, if $X$ is any (real) linear combination of two matrices $Y$ and $Z$ of the above form, we have $v^{*} \phi(X) v=0$.

ASSERTION 2. There exists a rank one orthogonal projection $X$ such that $\phi(X)$ is unitarily similar to [1] $\oplus O_{q} \oplus D_{1}$, where $q+1<k$ and $D_{1}$ is a diagonal matrix with diagonal entries in the interval $(0,1)$.

Proof. By Assertion 1, each $\phi\left(E_{j j}\right)$ has largest and smallest eigenvalues equal to 1 and 0 , respectively. Since $n<k \leq 2 n-2$ and

$$
k=\operatorname{tr} I_{k}=\operatorname{tr} \phi\left(I_{n}\right)=\sum_{j=1}^{n} \operatorname{tr} \phi\left(E_{j j}\right),
$$

we see that there exist at least two matrices $\phi\left(E_{j j}\right)$ with exactly one eigenvalue equal to 1 . If one of these matrices, say, $\phi\left(E_{j j}\right)$, is not an orthogonal projection in $\mathscr{H}_{k}$, then $E_{j j}$ is a desired matrix $X$. 
Suppose each matrix $\phi\left(E_{j j}\right)$ with one eigenvalue equal to 1 is an orthogonal projection, and $\phi\left(E_{11}\right)$ is one of them. Since $n<k$, by (2.4) again there exists $\phi\left(E_{p p}\right)$ with at least two eigenvalues equal to 1 . Without loss of generality, we may assume that $p=2$. By Assertion 1, there exists a unitary $U \in M_{k}$ such that

$$
\phi\left(E_{11}\right)=U\left(E_{11} \oplus O_{k-n}\right) U^{*} \quad \text { and } \quad \phi\left(E_{22}\right)=U\left([0] \oplus I_{r} \oplus C_{2}\right) U^{*}
$$

so that $r>1$ and $\left\|C_{2}\right\|<1$. For simplicity, assume that $U=I_{k}$; otherwise, replace $\phi$ by the mapping $X \mapsto U^{*} \phi(X) U$. So,

$$
\phi\left(E_{11}\right)=E_{11} \oplus O_{k-n} \quad \text { and } \quad \phi\left(E_{22}\right)=[0] \oplus I_{r} \oplus C_{2} .
$$

Let $Y_{1}=\phi\left(E_{11}+E_{22}\right), Y_{2}=\phi\left(E_{12}+E_{21}\right)$, and

$$
Y=\left(Y_{1}+Y_{2}\right) / 2=\phi\left(E_{11}+E_{22}+E_{12}+E_{21}\right) / 2 .
$$

Since $\|\phi(Z)\|=\|Z\|$, we have $1=\|Y\|=\left\|Y_{1}\right\|=\left\|Y_{2}\right\|$. Applying Assertion 1 to the matrices $\phi\left(E_{11}\right)$ and $Y_{2}$, and also to $\phi\left(E_{22}\right)$ and $Y_{2}$, we see that

$$
Y_{2}=\left(\begin{array}{ccc}
0 & u_{1}^{*} & u_{2}^{*} \\
u_{1} & O_{r} & * \\
u_{2} & * & *
\end{array}\right)
$$

for some $u_{1} \in \mathbb{C}^{r}$ and $u_{2} \in \mathbb{C}^{k-1-r}$. If $v \in \mathbb{C}^{k}$ is a unit vector so that $v^{*} Y v=1$, then

$$
2=2 v^{*} Y v=v^{*} Y_{1} v+v^{*} Y_{2} v \leq\left\|Y_{1}\right\|+\left\|Y_{2}\right\|=2,
$$

and hence $v^{*} Y_{1} v=1=v^{*} Y_{2} v$. Since $Y_{1}=I_{1+r} \oplus C_{2}$ with $\lambda_{1}\left(C_{2}\right)<1$ by (2.5), we see that $v \in \operatorname{span}\left\{e_{1}, \ldots, e_{1+r}\right\} \subseteq \mathbb{C}^{k}$. Thus, if $P$ is obtained from $I_{k}$ by taking its first $1+r$ columns, then $1=\left\|Y_{2}\right\|=v^{*} Y_{2} v \leq\left\|P^{*} Y_{2} P\right\| \leq\left\|Y_{2}\right\|$. It follows that

$$
1=\left\|P^{*} Y_{2} P\right\|=\left\|\left(\begin{array}{cc}
0 & u_{1}^{*} \\
u_{1} & O_{r}
\end{array}\right)\right\|
$$

thus, $u_{1}$ is a unit vector. Since $Y_{2}$ in the form (2.6) has norm 1 , we see that $u_{2}=0$ and there exists a unitary matrix $W=[1] \oplus W_{1} \oplus I_{k-1-r}$ such that $W Y_{2} W^{*}=\left(\begin{array}{ll}0 & 1 \\ 1 & 0\end{array}\right) \oplus Z_{2}$. Hence, $W\left(Y_{1}+Y_{2}\right) W^{*}=\left(\begin{array}{l}1 \\ 1 \\ 1\end{array}\right) \oplus Z_{0}$, and $Z_{0}$ is nonzero positive semidefinite such that

$$
\left\|Z_{0}\right\| \leq\left\|\left(\begin{array}{ll}
0 & 1 \\
1 & 1
\end{array}\right) \oplus Z_{0}\right\|=\left\|\phi\left(E_{22}+E_{12}+E_{21}\right)\right\|=\left\|E_{22}+E_{12}+E_{21}\right\|<2 .
$$

Thus, $Y$ is unitarily similar to the direct sum of a rank one orthogonal projection and a non-trivial $D$ with $0<\lambda_{1}(D)<1$. So, $X=\left(E_{11}+E_{22}+E_{12}+E_{21}\right) / 2$ is a desired rank one orthogonal projection. 
ASSERTION 3. There exist unitary $U \in M_{k}$ and $V \in M_{n}$ such that the mapping $\tilde{\phi}$ defined by

$$
X \mapsto U \phi\left(V X V^{*}\right) U^{*}
$$

satisfies

$$
\begin{array}{lll}
\tilde{\phi}(Y)=Y \oplus \tilde{f(Y)} & \text { for all } Y=[a] \oplus Y_{1}, & \text { or } \\
\tilde{\phi}(Y)=Y^{t} \oplus \tilde{f}(Y) & \text { for all } Y=[a] \oplus Y_{1}, &
\end{array}
$$

where $\tilde{f}: \mathscr{H}_{n} \rightarrow \mathscr{H}_{k-n}$ is a unital positive linear map satisfying $0<\left\|\tilde{f}\left(E_{11}\right)\right\|<1$.

Proof. By Assertion 2, we may replace $\phi$ by a mapping of the form (2.7) and assume that $\phi\left(E_{11}\right)=[1] \oplus O_{q} \oplus D_{1}$, where $q+1<k$ and $D_{1}$ is a diagonal matrix with diagonal entries in the interval $(0,1)$. Let $Y=[0] \oplus Y_{1} \in \mathscr{H}_{n}$, where $Y_{1} \in \mathscr{H}_{n-1},\left\|Y_{1}\right\|=1$. By Assertion 1 , the $(1,1)$ entry of $\phi(Y)$ is 0 . Since $\left\|\phi\left(E_{11}+Y\right)\right\|=\left\|E_{11}+Y\right\|=1$, the first row and column of $\phi(Y)$ are all zero. Therefore,

$$
\phi(Y)=\left(\begin{array}{ccc}
0 & 0 & 0 \\
0 & \psi\left(Y_{1}\right) & * \\
0 & * & *
\end{array}\right) \text { with } \psi\left(Y_{1}\right) \in \mathscr{H}_{q}
$$

Since $1=\|\phi(Y)\|$, there exists a unit vector $v$ such that $1=\left|v^{*} \phi(Y) v\right|=\|\phi(Y)\|$. Clearly, the first entry of $v$ must be zero. Suppose $v=\left[\begin{array}{c}0 \\ v_{1} \\ v_{2}\end{array}\right]$ with $v_{1} \in \mathbb{C}^{q}$ and $v_{2} \in \mathbb{C}^{k-1-q}$. Since $\phi\left(E_{11}\right)=[1] \oplus O_{q} \oplus D_{1}$ and

$$
\left|v_{2}^{*} D_{1} v_{2} \pm v^{*} \phi(Y) v\right|=\left|v^{*} \phi\left(E_{11} \pm Y\right) v\right| \leq\left\|E_{11} \pm Y\right\|=1,
$$

we see that $v_{2}=0$ and $\left|v_{1}^{*} \psi\left(Y_{1}\right) v_{1}\right|=1=\left\|Y_{1}\right\|$. Hence, the mapping from $M_{n-1}$ to $M_{q}$ defined by $Y_{1} \mapsto \psi\left(Y_{1}\right)$ is unital and satisfies $\left\|\psi\left(Y_{1}\right)\right\|=\left\|Y_{1}\right\|$ for all $Y_{1} \in \mathscr{H}_{n-1}$. Since $q \leq k-2 \leq 2 n-4$, we can apply induction assumption to $\psi$ and conclude that $\psi$ on $\mathscr{H}_{n-1}$ has the standard form:

$$
Y_{1} \mapsto U^{*}\left[Y_{1} \oplus f\left(Y_{1}\right)\right] U \quad \text { or } \quad Y_{1} \mapsto U^{*}\left[Y_{1}^{t} \oplus f\left(Y_{1}\right)\right] U
$$

for some unitary $U \in M_{q}$. Now, the mapping $\tilde{\phi}$ defined by

$$
X \mapsto\left([1] \oplus U \oplus I_{k-q-1}\right) \phi(X)\left([1] \oplus U^{*} \oplus I_{k-q-1}\right)
$$

satisfies (2.8) or (2.9). 
Proof of THE Claim. By Assertion 3, we can modify $\phi$ to $\tilde{\phi}$ that satisfies (2.8) or (2.9), where $\tilde{f}$ is a unital linear map satisfying $0<\left\|\tilde{f}\left(E_{11}\right)\right\|<1$. We may further assume that $\tilde{\phi}$ satisfies (2.8); otherwise, replace $\phi$ by the mapping $A \mapsto \phi\left(A^{t}\right)$. For simplicity, we assume that $\phi=\tilde{\phi}$.

To prove the claim, we note that every matrix in $\mathscr{H}_{n}$ is a linear combination of rank one orthogonal projections. Therefore, we only need to show that if $X \in \mathscr{H}_{n}$ is a rank one orthogonal projection, then

$$
\phi(X)=g(X) \oplus \tilde{f}(X),
$$

where $g(X)$ is a rank one $n \times n$ orthogonal projection.

If $X=E_{11}$ or $X$ has the form [0] $\oplus X_{1}$, then we are done because $\phi=\tilde{\phi}$ satisfies (2.8). Now, suppose $X$ is not of these forms. Then $X=u u^{*}$, where $u=a e_{1}+b v \in \mathbb{C}^{n}$ is a unit vector such that $v \in e_{1}^{\perp}$ and $a, b$ are nonzero complex numbers satisfying $|a|^{2}+|b|^{2}=1$. Replacing $u$ by $\xi_{1} u$ for a suitable complex unit $\xi_{1}$, we may assume that $a>0$; then replacing $v$ by $\xi_{2} v$ for a suitable complex unit $\xi_{2}$, we may assume that $b>0$ as well. So, $(a, b)=(\cos \theta, \sin \theta)$ for some $\theta \in(0, \pi / 2)$. Suppose $V \in M_{n}$ is a unitary matrix with $e_{1}$ and $v$ as the first two columns. Then $V$ has the form [1] $\oplus V_{1}$ and satisfies

$$
V^{*} X V=\cos ^{2} \theta E_{11}+\cos \theta \sin \theta\left(E_{12}+E_{21}\right)+\sin ^{2} \theta E_{22} .
$$

Consider the mapping $\phi_{V}$ defined by $A \mapsto\left(V^{*} \oplus I_{k-n}\right) \phi\left(V A V^{*}\right)\left(V \oplus I_{k-n}\right)$. Note that the mapping $\phi_{V}$ inherits all the properties we have established in Assertions 1-3, (2.8) for $\phi$. Moreover, if we can show that $\phi_{V}$ sends the matrix

$$
\cos ^{2} \theta E_{11}+\cos \theta \sin \theta\left(E_{12}+E_{21}\right)+\sin ^{2} \theta E_{22}
$$

to a matrix of the form $Z_{1} \oplus Z_{2}$ so that $Z_{1} \in M_{n}$ is a rank one orthogonal projection, then $\phi(X)=\left(V \oplus I_{k-n}\right) \phi_{V}\left(V^{*} X V\right)\left(V^{*} \oplus I_{k-n}\right)=V Z_{1} V^{*} \oplus Z_{2}$, where $V Z_{1} V^{*}$ is a rank one orthogonal projection as desired. So, we focus on $\phi_{V}$. For simplicity, we write $\phi_{V}$ as $\phi$ in the rest of our proof. For $j \in\{1, \ldots, n\}$, let $\phi\left(E_{j j}\right)=E_{j j} \oplus C_{j}$. Then $C_{1}=\tilde{f}\left(E_{11}\right)$ satisfies $0<\left\|C_{1}\right\|<1$ and

$$
C_{1}+\cdots+C_{n}=I_{k-n} .
$$

We consider two cases.

Case 1. Suppose $\lambda_{1}\left(C_{1}+C_{2}\right)<1$, that is, $\phi\left(E_{11}+E_{22}\right)$ only has two eigenvalues equal to 1 . If $v \in \mathbb{C}^{k}$ satisfies $v^{*}\left(\phi\left(E_{11}+E_{22}\right)\right) v=1$, then only the first two entries of $v$ can be nonzero. Now,

$$
\begin{aligned}
2 & =\left\|\left(e_{1}+e_{2}\right)\left(e_{1}+e_{2}\right)^{*}\right\|=\left\|\phi\left(\left(e_{1}+e_{2}\right)\left(e_{1}+e_{2}\right)^{*}\right)\right\| \\
& \leq\left\|\phi\left(e_{1} e_{1}^{*}+e_{2} e_{2}^{*}\right)\right\|+\left\|\phi\left(e_{1} e_{2}^{*}+e_{2} e_{1}^{*}\right)\right\|=2 .
\end{aligned}
$$


So, there is a unit vector $v \in \mathbb{C}^{n}$ such that

$$
v^{*}\left(\phi\left(e_{1} e_{1}^{*}+e_{2} e_{2}^{*}\right)\right) v=1=v^{*}\left(\phi\left(e_{1} e_{2}^{*}+e_{2} e_{1}^{*}\right)\right) v .
$$

Thus, the leading $2 \times 2$ principal submatrix of $\phi\left(e_{1} e_{2}^{*}+e_{2} e_{1}^{*}\right)$ has norm one. By Assertion 1 , the $(1,1)$ and $(2,2)$ entries of $\phi\left(e_{1} e_{2}^{*}+e_{2} e_{1}^{*}\right)$ are zero. Hence, there is a complex unit $\mu$ such that $\phi\left(e_{1} e_{2}^{*}+e_{2} e_{1}^{*}\right)=\left(\mu e_{1} e_{2}^{*}+\bar{\mu} e_{2} e_{1}^{*}\right) \oplus D$. Therefore,

$$
\phi\left(\left(\cos \theta e_{1}+\sin \theta e_{2}\right)\left(\cos \theta e_{1}+\sin \theta e_{2}\right)^{*}\right)=\left(\begin{array}{cc}
\cos ^{2} \theta & \mu \cos \theta \sin \theta \\
\bar{\mu} \cos \theta \sin \theta & \sin ^{2} \theta
\end{array}\right) \oplus \tilde{D} .
$$

Since

$$
\left\|\phi\left(\left(\cos \theta e_{1}+\sin \theta e_{2}\right)\left(\cos \theta e_{1}+\sin \theta e_{2}\right)^{*} \pm \sum_{j=3}^{n} e_{j} e_{j}^{*}\right)\right\|=1,
$$

we see that $\tilde{D}$ has the form $O_{n-2} \oplus \hat{D}$. Hence, $\phi\left(\left(\cos \theta e_{1}+\sin \theta e_{2}\right)\left(\cos \theta e_{1}+\sin \theta e_{2}\right)^{*}\right)$ has the desired form (2.10).

Case 2. Suppose $\lambda_{1}\left(C_{1}+C_{2}\right)=1$. We shall prove that there exists a sequence of unit vectors $\left\{v_{r}\right\}$ in the linear span of $\left\{e_{2}, \ldots, e_{n}\right\} \subseteq \mathbb{C}^{n}$ such that $v_{r} \rightarrow e_{2}$, and for each $r$, $\phi\left(E_{11}+v_{r} v_{r}^{*}\right)$ has only two eigenvalues equal to 1 . By the result in Case 1,

$$
\phi\left(\left(\cos \theta e_{1}+\sin \theta v_{r}\right)\left(\cos \theta e_{1}+\sin \theta v_{r}\right)^{*}\right)
$$

has the desired form (2.10). By continuity, we see that

$$
\phi\left(\left(\cos \theta e_{1}+\sin \theta e_{2}\right)\left(\cos \theta e_{1}+\sin \theta e_{2}\right)^{*}\right)
$$

has the desired form (2.10) as well.

To construct our sequence $\left\{v_{r}\right\}$, note that by (2.11) and the fact that $0<\left\|C_{1}\right\|<1$, we have

$$
\left(I_{k-n}-C_{1}\right)^{-1 / 2}\left(C_{2}+\cdots+C_{n}\right)\left(I_{k-n}-C_{1}\right)^{-1 / 2}=I_{k-n} .
$$

Since $k-n \leq n-2$, comparing traces, we see that there exists $j \geq 3$ such that

$$
\left(I_{k-n}-C_{1}\right)^{-1 / 2} C_{j}\left(I_{k-n}-C_{1}\right)^{-1 / 2}
$$

is a strict contraction, equivalently, $\lambda_{1}\left(C_{1}+C_{j}\right)<1$. Without loss of generality, we may assume that $j=3$. Let $\phi\left(E_{23}+E_{32}\right)=\left(E_{23}+E_{32}\right) \oplus C_{23}$. For $t \in[0, \pi / 2]$, let

$$
F(t)=v(t) v(t)^{*} \quad \text { with } \quad v(t)=\cos t e_{2}+\sin t e_{3} \in \mathbb{C}^{n}
$$

Then

$$
\phi\left(E_{11}+F(t)\right)=\left[E_{11}+F(t)\right] \oplus\left[C_{1}+\cos ^{2} t C_{2}+\sin ^{2} t C_{3}+\cos t \sin t C_{23}\right] .
$$


If $\phi\left(E_{11}+F(t)\right)$ has more than two eigenvalues equal 1 , then

$$
\begin{aligned}
0 & =\operatorname{det}\left(I_{k-n}-\left(C_{1}+\cos ^{2} t C_{2}+\sin ^{2} t C_{3}+\cos t \sin t C_{23}\right)\right) \\
& =\operatorname{det}\left(\cos ^{2} t\left[\left(1+\tan ^{2} t\right)\left(I_{k-n}-C_{1}\right)-C_{2}-\tan ^{2} t C_{3}-\tan t C_{23}\right]\right) \\
& =\cos ^{2(k-n)} t \operatorname{det}\left(\left(I_{k-n}-C_{1}-C_{2}\right)-\tan t C_{23}+\tan ^{2} t\left(I_{k-n}-C_{1}-C_{3}\right)\right)
\end{aligned}
$$

Since $\phi\left(E_{11}+E_{33}\right)$ has only two eigenvalues equal to $1, \operatorname{det}\left(I_{k-n}-C_{1}-C_{3}\right) \neq 0$. It follows that (2.12) only has finitely many roots in the interval $[0, \pi / 2]$. Thus, we can find a sequence $\left\{t_{r}\right\} \rightarrow 0$ such that $\left\{v_{r}\right\}=\left\{v\left(t_{r}\right)\right\} \rightarrow e_{2}$, and for each $r, E_{1\}}+v_{r} v_{r}^{*}$ has only two eigenvalues equal to 1 as desired.

\section{Proof of the main theorems}

PROOF OF THEOREM 1.2. The 'if' part of the theorem is clear. Suppose $k \leq 2 n-2$, $\phi\left(I_{n}\right)=I_{k}$ and $\|\phi(X)\|=\|X\|$ for all essentially Hermitian $X$. By Theorem 2.1, $\phi(X)$ is Hermitian whenever $X$ is Hermitian. Now, the result follows from Theorem 2.3.

For the last statement, suppose $n \geq 2$ and $k \geq 2 n-1$. Let

$$
W=\left(\begin{array}{cccc}
1 / \sqrt{2} & 0 & 1 / \sqrt{2} & 0 \\
0 & I_{n-1} & 0 & 0 \\
0 & 0 & 0 & I_{n-1}
\end{array}\right)
$$

Define $\phi: M_{n} \rightarrow M_{k}$ by

$$
\begin{aligned}
\phi(A) & =W\left[A \oplus A^{t}\right] W^{*} \oplus(\operatorname{tr} A / n) I_{k-2 n+1} \\
& =\left(\begin{array}{ccc}
A_{11} & A_{12} / \sqrt{2} & A_{21}^{t} / \sqrt{2} \\
A_{21} / \sqrt{2} & A_{22} & 0 \\
A_{12}^{t} / \sqrt{2} & 0 & A_{22}^{t}
\end{array}\right) \oplus(\operatorname{tr} A / n) I_{k-2 n+1}
\end{aligned}
$$

for any

$$
A=\left(\begin{array}{ll}
A_{11} & A_{12} \\
A_{21} & A_{22}
\end{array}\right) \quad \text { with } A_{22} \in M_{n-1}
$$

Since $W W^{*}=I_{2 n-1}$, by the interlacing inequalities for eigenvalues of Hermitian matrices [4, Theorem 4.3.6], if $A \in \mathscr{H}_{n}$ and $B=W\left[A \oplus A^{t}\right] W^{*}$, then $\lambda_{1}(B)=\lambda_{1}(A)$ and $\lambda_{2 n-1}(B)=\lambda_{n}(A)$. Consequently, $\|\phi(X)\|=\|X\|$ for all essentially Hermitian $X \in M_{n}$.

If $\phi$ has the standard form (1.2), then there exist a contractive linear map $f: M_{n} \rightarrow$ $M_{k-n}$ and a unitary matrix $U \in M_{k}$ such that $U \phi(A)=\left(A^{\dagger} \oplus f(A)\right) U$, where $A^{\dagger}=A$ 
or $A^{t}$. Partition $U$ into $U=\left(U_{i j}\right)_{i, j=1}^{2}$, where $U_{22} \in M_{k-1}$, then we have

$$
\begin{gathered}
\left(\begin{array}{ll}
U_{11} & U_{12} \\
U_{21} & U_{22}
\end{array}\right)\left(\begin{array}{cccc}
A_{11} & A_{12} / \sqrt{2} & A_{21}^{t} / \sqrt{2} & 0 \\
A_{21} / \sqrt{2} & A_{22} & 0 & 0 \\
A_{12}^{t} / \sqrt{2} & 0 & A_{22}^{t} & 0 \\
0 & 0 & 0 & (\operatorname{tr} A / n) I_{k-2 n+1}
\end{array}\right) \\
=\left(\begin{array}{cc}
A^{+} & 0 \\
0 & f(A)
\end{array}\right)\left(\begin{array}{cc}
U_{11} & U_{12} \\
U_{21} & U_{22}
\end{array}\right) .
\end{gathered}
$$

Let $A=E_{11}$ and consider the first row on both sides. We have $\left(\begin{array}{ll}U_{11} & 0\end{array}\right)=\left(\begin{array}{ll}U_{11} & U_{12}\end{array}\right)$. Hence, $U_{11}=e^{i \theta}$ for some real number $\theta$ and $U_{12}, U_{21}$ are both zero. Consider the first row on both sides in the general case, we have

$$
e^{i \theta}\left(\begin{array}{llll}
A_{11} & A_{12} / \sqrt{2} & A_{21}^{t} / \sqrt{2} & 0
\end{array}\right)=\left(\begin{array}{ll}
A_{12}^{\dagger} & 0
\end{array}\right) U_{22},
$$

for all $A \in M_{n}$, which is impossible. Hence, $\phi$ is not of the standard form (1.2).

Proof OF THEOREM 1.1. Suppose $k \leq 2 n-1$, and $\|\phi(X)\|=\|X\|$ for all $X \in M_{n}$. Clearly, we have $k \geq n$. If $k=n$ then (1.1) follows from Kadison's result [6]. So we may assume that $n<k \leq 2 n-1$. By the result in [1], it is impossible that $\phi(U)$ is unitary for every unitary $U \in M_{n}$. Thus, there exists a unitary $X \in M_{n}$ such that $\phi(X)$ is not unitary. By replacing $\phi$ with the map $A \mapsto \phi(X A)$, if necessary, we may assume that $X=I$. Therefore, $\phi$ satisfies all conditions in Theorem 2.1 with $1 \leq p \leq 2 n-2$. Let $U, V \in M_{k}$, and $\psi$ be as given by Theorem 2.1. Then $\psi$ satisfies the conditions for Theorem 1.2 (with $\phi, k$ replaced by $\psi, p$ ). So, there exists a unitary $W_{1} \in M_{p}$ and a unital positive linear map $\tilde{f}: M_{n} \rightarrow M_{p-n}$ such that $\psi$ has the form

$$
A \mapsto W_{1}\left[A^{\dagger} \oplus \tilde{f}(A)\right] W_{1}^{*},
$$

where $A^{+}=A$ or $A^{\prime}$. Let $W=W_{1} \oplus I_{k-p}$. Then the mapping $\phi_{0}: M_{n} \rightarrow M_{k}$ defined by $A \mapsto W^{*} U^{*} \phi(A) V^{*} W$ has the form

$$
A \mapsto\left(\begin{array}{ccc}
A^{\dagger} & 0 & * \\
0 & \tilde{f(A)} & * \\
* & * & g(A)
\end{array}\right) .
$$

If $A \in M_{n}$ is unitary, then $\left\|\phi_{0}(A)\right\|=\|A\|$ implies that

$$
\phi_{0}(A)=A^{\dagger} \oplus\left(\begin{array}{cc}
\tilde{f}(A) & * \\
* & g(A)
\end{array}\right) .
$$

Since this is true for $n^{2}$ linearly independent unitary matrices $A$, it follows that (3.1) holds for any $A \in M_{n}$. Consequently, the original map $\phi$ has the form (1.1) as asserted. 
For the last statement, suppose $n \geq 2$ and $k \geq 2 n$. Let

$$
W=\left(\begin{array}{cccc}
1 / \sqrt{2} & 0 & 1 / \sqrt{2} & 0 \\
0 & I_{n-1} & 0 & 0 \\
0 & 0 & 0 & I_{n-1} \\
1 / \sqrt{2} & 0 & -1 / \sqrt{2} & 0
\end{array}\right) \quad \text { and } \quad P=I_{2 n-1} \oplus O_{k-2 n+1}
$$

Define $\phi: M_{n} \rightarrow M_{k}$ by

$$
\begin{aligned}
\phi(A) & =P\left(W \oplus I_{k-2 n}\right)\left[A \oplus A^{t} \oplus O_{k-2 n}\right]\left(W \oplus I_{k-2 n}\right)^{*} \\
& =\left(\begin{array}{ccccc}
A_{11} & A_{12} / \sqrt{2} & A_{21}^{t} / \sqrt{2} & 0 & 0 \\
A_{21} / \sqrt{2} & A_{22} & 0 & A_{21} / \sqrt{2} & 0 \\
A_{12}^{t} / \sqrt{2} & 0 & A_{22}^{t} & -A_{12}^{t} / \sqrt{2} & 0 \\
0 & 0 & 0 & 0 & 0 \\
0 & 0 & 0 & 0 & 0
\end{array}\right)
\end{aligned}
$$

for any

$$
A=\left(\begin{array}{ll}
A_{11} & A_{12} \\
A_{21} & A_{22}
\end{array}\right) \text { with } A_{22} \in M_{n-1} .
$$

Note that $\phi(A) \phi(A)^{*}=B \oplus O_{k-2 n+1}$, where $B \in \mathscr{H}_{2 n-1}$ is a leading principal submatrix of $W\left(A A^{*} \oplus\left(A A^{*}\right)^{l}\right) W^{*}$. By the interlacing inequalities for eigenvalues of Hermitian matrices [4, Theorem 4.3.6], we have $\|\phi(A)\|=\|A\|$. By an argument similar to the one in the proof of Theorem 1.2, we can show that $\phi$ is not of the form (1.1).

\section{Related results and questions}

Motivated by Theorem 1.1 and the example constructed in its proof, we have the following.

Proposition 4.1. Suppose $P$ and $Q$ are $n(p+q) \times m$ matrices such that

$$
I-P P^{*} \geq 0, \quad I-Q Q^{*} \geq 0,
$$

and $\operatorname{rank}\left(I-P P^{*}\right)+\operatorname{rank}\left(I-Q Q^{*}\right)<p+q$. Let $W_{1}, W_{2} \in M_{k}$ be unitary, and let $f: M_{n} \rightarrow M_{k-m}$ be a contractive linear map. If $\phi: M_{n} \rightarrow M_{k}$ is defined by

$$
\phi(X)=W_{1}\left\{P^{*}\left[\left(X \otimes I_{p}\right) \oplus\left(X^{i} \otimes I_{q}\right)\right] Q \oplus f(X)\right\} W_{2},
$$

then $\|\phi(X)\|=\|X\|$ for all $X \in M_{n}$. 
Proof. It is clear that $\|\phi(X)\| \leq\|X\|$. To prove the reverse inequality, suppose $P$ has singular value decomposition $U D V$, where $U \in M_{n(p+q)}$ and $V \in M_{m}$ are unitary, and the singular values of $P$ lie in the $(1,1),(2,2), \ldots$ positions of $D$ in descending order. Let $\tilde{D}$ be obtained from $D$ by setting all the entries in $(0,1)$ to 0 , and let $\tilde{P}=U \tilde{D} V$. Apply a similar construction to $Q$ to get $\tilde{Q}$. Then

$$
\begin{aligned}
& \operatorname{rank}\left(I-\tilde{P} \tilde{P}^{*}\right)+\operatorname{rank}\left(I-\tilde{Q} \tilde{Q}^{*}\right) \\
& \quad=\operatorname{rank}\left(I-P P^{*}\right)+\operatorname{rank}\left(I-Q Q^{*}\right)<p+q .
\end{aligned}
$$

If the largest singular value of $X$ is $s_{1}=\|X\|$, then $s_{1}$ is a singular value of $\left(X \otimes I_{p}\right) \oplus$ $\left(X^{\prime} \otimes I_{q}\right)$ with multiplicity at least $p+q$. By (4.1) and a result of Thompson [7], the matrix $\tilde{P}^{*}\left[\left(X \otimes I_{p}\right) \oplus\left(X^{t} \otimes I_{q}\right)\right] \tilde{Q}$ has largest singular value equal to $s_{1}$ also. Thus, we have $\|X\|=s_{1}=\left\|\tilde{P}^{*}\left[\left(X \otimes I_{p}\right) \oplus\left(X^{t} \otimes I_{q}\right)\right] \tilde{Q}\right\| \leq\|\phi(X)\|$.

Using a similar argument as in the proof of Proposition 4.1 and the interlacing inequalities on Hermitian matrices (see [4, Theorem 4.3.6]), we have the following.

Proposition 4.2. Suppose $P$ is an $n(p+q) \times m$ matrix such that $P^{*} P=I_{m}$, where $0 \leq n(p+q)-m<p+q$. Let $U \in M_{k}$ be unitary, and $f: M_{n} \rightarrow M_{k-m}$ be a unital positive linear map. If $\phi: M_{n} \rightarrow M_{k}$ is defined by

$$
\phi(X)=U^{*}\left\{P^{*}\left[\left(X \otimes I_{p}\right) \oplus\left(X^{i} \otimes I_{q}\right)\right] P \oplus f(X)\right\} U,
$$

then $\|\phi(X)\|=\|X\|$ for all essentially Hermitian $X \in M_{n}$.

Recall that the numerical range of a matrix $A \in M_{n}$ is the set

$$
W(A)=\left\{x^{*} A x: x \in \mathbb{C}^{n}, x^{*} x=1\right\},
$$

which is a useful concept in matrix and operator theory, and has been studied extensively; see [5, Chapter 1]. We have the following proposition.

Proposition 4.3. Let $V_{m}=M_{m}$ or $\mathscr{H}_{m}$. Suppose $\phi: V_{n} \rightarrow V_{k}$ is linear. If $\phi$ has the form given in Proposition 4.2, then

$$
W(\phi(X))=W(X) \text { for all } X \in V_{n} .
$$

When $k \leq 2 n-2,(4.2)$ holds if and only if $\phi$ has the form (1.2) in Theorem 1.2.

Proof. Suppose $\phi$ has the form in Proposition 4.2. If $X \in \mathscr{H}_{n}$, then $X$ and $\phi(X)$ have the same largest and smallest eigenvalues; since $W(X)$ is the convex hull of the largest and smallest eigenvalues of $X$, it follows that $W(\phi(X))=W(X)$. 
Suppose $V_{n}=M_{n}$ and $X \in M_{n}$ is not Hermitian. Then $X=H+i G$ for some Hermitian $H$ and $G$. Now, $\phi(\cos t H+\sin t G)$ and $\cos t H+\sin t G$ have the same largest and smallest eigenvalues for all $t \in[0,2 \pi)$, we see that the two convex sets $W(X)$ and $W(\phi(X))$ have the same support lines; see [5, Theorem 1.5.11]. Thus, the two sets are equal.

Suppose $k \leq 2 n-2$. If $V_{n}=\mathscr{H}_{n}$, the result follows readily from Theorem 2.3. If $V_{n}=M_{n}$, one can use the fact that $W(X) \subseteq \mathbb{R}$ if and only if $X$ is Hermitian to conclude that $\phi\left(\mathscr{H}_{n}\right) \subseteq \mathscr{H}_{k}$. Then the result follows from the Hermitian case.

There are several related problems that deserve further investigation.

(1) If $\phi: M_{n} \rightarrow M_{k}$ has the form in Proposition 4.1, then $\|\phi(A)\|=\|A\|$ for all $A \in M_{n}$. It would be nice to know whether the converse is true.

(2) If $\phi: M_{n} \rightarrow M_{k}$ has the form in Proposition 4.2, then $\|\phi(A)\|=\|A\|$ for all essentially Hermitian $A \in M_{n}$. It would be nice to know whether the converse is true. Note that by Theorem 2.1 and Theorem 2.3 (b), the problem is equivalent to studying positive linear maps $\phi$ such that $A$ is positive definite whenever $\phi(A)$ is positive definite.

(3) One can ask whether the converse of the first statement in Proposition 4.3 is true.

(4) One can study the above problems under the additional assumption that $\phi$ is a decomposable or completely positive linear map.

\section{Acknowledgment}

We thank the referee for some helpful suggestions. Also, we thank Professor Ngai-Ching Wong for helpful comments and for drawing our attention to the problem, which was motivated by the work in [2].

The second author would like to thank Professors Leiba Rodman and Peter Šemrl for helpful discussion on linear maps between matrix algebras preserving numerical ranges.

Research of the first author was supported by a PIMS Postdoctoral Fellowship, and the research of the second author was partially supported by NSF.

\section{References}

[1] W. S. Cheung and C. K. Li, 'Linear maps transforming the unitary group', Canad. Math. Bulletin 46 (2003), 54-58.

[2] C.-H. Chu and N.-C. Wong, 'Isometries between $C^{*}$-algebras', Revista Matematica Iberoamericana (to appear).

[3] J. W. Helton and L. Rodman, 'Signature preserving linear maps on Hermitian matrices', Linear and Multilinear Algebra 17 (1985), 29-37. 
[4] R. Horn and C. Johnson, Matrix analysis (Cambridge University Press, Cambridge, 1985).

[5] _ Topics in matrix analysis (Cambridge University Press, Cambridge, 1991).

[6] R. V. Kadison, 'Isometries of operator algebras', Ann. of Math. 54 (1951), 325-338.

[7] R. C. Thompson, 'Principal submatrices. IX. Interlacing inequalities for singular values of submatrices', Linear Algebra Appl. 5 (1972), 1-12.

Center of Linear Algebra and Combinatorics

University of Lisbon

Lisbon

Portugal

(Current address:

Department of Mathematics

University of Hong Kong

Hong Kong)

e-mail: a9000068@yahoo.com

Department of Mathematics

Iowa State University

Ames

Iowa 50011

USA

e-mail: ytpoon@iastate.edu
Department of Mathematics

College of William and Mary P.O. Box 8795 Williamsburg Virginia 23187-8795 USA

e-mail: ckli@math.wm.edu 